DOI: $10.52119 /$ LPHS. 2020.81.10.003

Владимир Попов 1

К ПРОБЛЕМЕ РАСШИРЕНИЯ МАТРИЧНОЙ СЕМАНТИКИ, АДЕКВАТНОЙ КЛАССИЧЕСКОЙ ИМПЛИКАТИВНОЙ ЛОГИКЕ, ДО МАТРИЧНОЙ СЕМАНТИКИ, АДЕКВАТНОЙ КЛАССИЧЕСКОЙ ИМПЛИКАТИВНО-НЕГАТИВНОЙ ЛОГИКЕ (ЧАСТЬ 2)

Аннотация. Эта работа продолжает представленные в (Попов 2019а) исследования проблемы расширения семантики, адекватной собственному фрагменту логики, до семантики, адекватной этой логике. В предлагаемой статье мы используем результаты, полученные в работах (Попов 2019а) и (Попов 2019b), и опираемся на определения, соглашения и замечания из этих работ. Основное содержание статьи размещено в двух разделах (первый раздел и второй раздел). В первом разделе изучается вопрос о возможности построения логической матрицы вида $\langle M(1 / 2,1,0,1 / 2), f\rangle$ (где $f$ есть унарная операция на множестве $\{1,1 / 2,0\})$, адекватной классической импликативно-негативной логике $C l_{\supset \neg}$. Заметим, что $M(1 / 2,1,0,1 / 2)$ принадлежит пересчету всех адекватных классической импликативной логике $C l_{\supset}$ трехзначных логических матриц, носителем каждой из которых является множество $\{1,1 / 2,0\}$, а выделенным множеством каждой из которых является множество $\{1\}$ (см. Попов 2019b). Здесь мы даем отрицательный ответ на указанный вопрос. Центральный вопрос второго раздела - вопрос о возможности построения логической матрицы вида $\langle M(1 / 2,0,0,1 / 2), f\rangle$ (где $f$ есть унарная операция на множестве $\{1,1 / 2,0\}$ ), адекватной классической импликативно-негативной логике $C l_{\supset \neg}$. Заметим, что $M(1 / 2,0,0,1 / 2)$ принадлежит пересчету всех адекватных классической импликативной логике $C l_{\supset}$ трехзначных логических матриц, носителем каждой из которых является множество $\{1,1 / 2,0\}$, а выделенным множеством каждой из которых является множество $\{1\}$ (см. Попов 2019b). Мы даем отрицательный ответ на центральный вопрос второго раздела и вносим исправление в анонс, сделанный в (Попов 2019а).

Ключевые слова: трехзначная логическая матрица с одним выделенным значением, $L_{\supset \neg^{-}}$

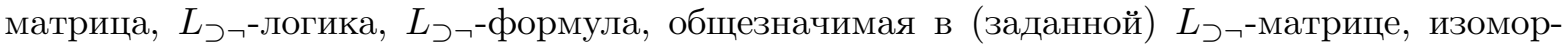
физм логических матриц.

\footnotetext{
${ }^{1}$ Попов Владимир Михайлович - кандидат философских наук, доцент кафедры логики философского факультета МГУ имени М. В. Ломоносова.

Vladimir Popov, PhD, associate professor, Department of Logic, Faculty of Philosophy, Lomonosov Moscow State University. pphiloslog@mail.ru

${ }^{2}$ Работа выполнена при поддержке РФФИ, проект № 19-011-00536 А.
} 
ЛОГИКА СЕГОДНЯ

\section{Vladimir Popov \\ ON THE PROBLEM OF EXPANSION OF MATRIX SEMANTICS ADEQUATE TO CLASSICAL IMPLICATIVE LOGIC TO MATRIX SEMANTICS ADEQUATE TO CLASSICAL IMPLICATIVE-NEGATIVE LOGIC (PART 2)}

Abstract. This work continues the studies presented in (Popov 2019a) of the problem of expansion of a semantics adequate to a proper fragment of a logic, to semantics which is adequate to this logic. In the present article, we rely on the results obtained in (Popov 2019a) and (Popov $2019 \mathrm{~b}$ ), and rely on definitions, agreements, and comments from these works. The main content of the article is placed in two sections (the first section and the second section). In the first section, we study the question of the possibility of constructing a logical matrix of the form $\langle M(1 / 2,1,0,1 / 2), f\rangle$ (where $f$ is a unary operation on the set $\{1,1 / 2,0\}$ ), adequate to the classical implicative-negative logic $C l_{\supset \neg}$. Note that $M(1 / 2,1,0,1 / 2)$ belongs to the set of all three-valued logical matrices adequate to the classical implicative logic $C l_{\supset}$, each of which is supported by the set $\{1,1 / 2,0\}$, and the designated set of each of which is the set $\{1\}$ (see Popov 2019b). Here we give a negative answer to this question. The central question of the second section is the question of the possibility of constructing a logical matrix of the form $\langle M(1 / 2,0,0,1 / 2), f\rangle$ (where $f$ is a unary operation on the set $\{1,1 / 2,0\}$ ), adequate to the classical implicative- negative logic $C l_{\supset \neg}$. Note that $M(1 / 2,0,0,1 / 2)$ belongs to the set of all three-valued logical matrices adequate to the classical implicative logic $C l_{\supset}$, each of which is supported by the set $\{1,1 / 2,0\}$, and the designated set of each of which is the set $\{1\}$ (see Popov 2019b). We give a negative answer to the central question of the second section and make corrections to the announcement made in (Popov 2019a).

Keywords: three-valued logical matrix with one designated value, $L_{\supset \neg^{-}}$matrix, $L_{\supset \neg^{-}}$-logic, $L_{\supset \neg^{-}}$ formula, which is valid in a (given) $L_{\supset \neg}$-matrix, isomorphism of logical matrices.

\section{Первый раздел}

В этом разделе мы используем определенное в (Попов 2019а) понятие регуляр-

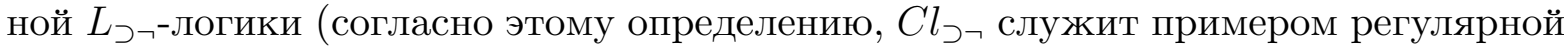
$L_{\supset \neg}$-логики) и доказываем, что для всякой унарной оерации $f$ на носителе $L_{\supset \neg^{-}}$ матрицы $M(1 / 2,1,0,1 / 2)$ упорядоченная пара $\langle M(1 / 2,1,0,1 / 2), f\rangle$ есть такая $L_{\supset}-$ матрица, что множество всех, общезначимых в $\langle M(1 / 2,1,0,1 / 2), f\rangle L_{\supset \neg}$-формул не является регулярной $L_{\supset \neg}$-логикой (в частности, не является $L_{\supset \neg}$-логикой $C l_{\supset \neg}$ ).

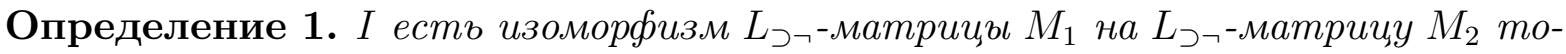

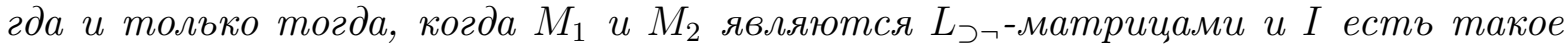

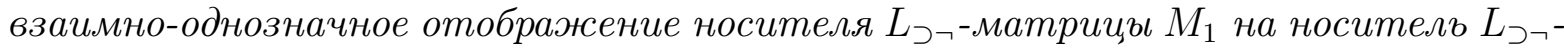
матрищы $M_{2}$, что выполняются следующие три условия: (1) I отображает вы-

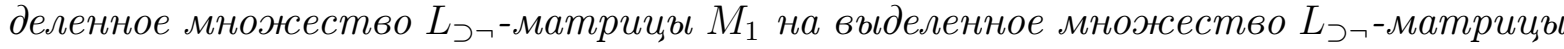


Владимир Попов. К проблеме расширения матричной семантики...

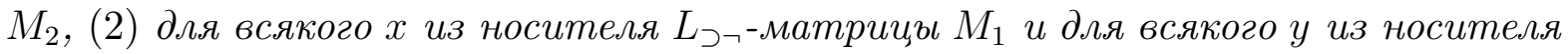

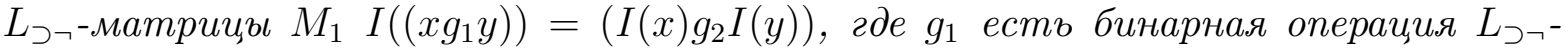
матрицы $M_{1}$, а g $g_{2}$ есть бинарная операция $L_{\supset}$-матричъ $M_{2}$, (3) для всякого $x$

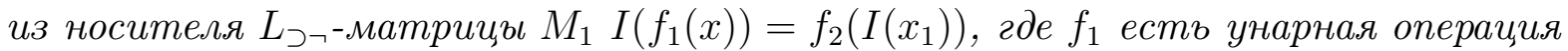

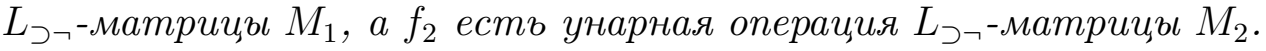

В свете этого определения очевидна справедливость следующего замечания 1.

Замечание 1. Если $\left\langle\{1,1 / 2,0\},\{1\}, g_{1}, f_{1}\right\rangle u\left\langle\{1,1 / 2,0\},\{1\}, g_{2}, f_{2}\right\rangle$ являются $L_{\supset \neg^{-}}$

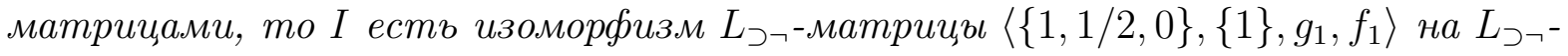
матричу $\left\langle\{1,1 / 2,0\},\{1\}, g_{2}, f_{2}\right\rangle$ тогда и только тогда, когда I есть такое взаимнооднозначное отображение множества $\{1,1 / 2,0\}$ на себя, что выполняются следующие три условия: (1) $I(1)=1$, (2) для всякого х из $\{1,1 / 2,0\}$ и для всякого у из $\{1,1 / 2,0\} I\left(\left(x g_{1} y\right)\right)=\left(I(x) g_{2} I(y)\right)$, (3) для всякого $x$ из $\{1,1 / 2,0\} I\left(f_{1}(x)\right)=$ $f_{2}(I(x))$.

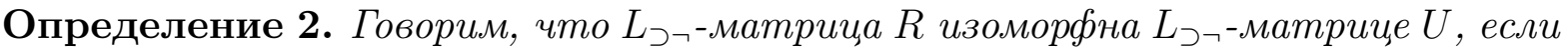

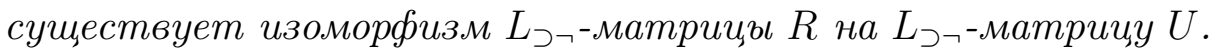

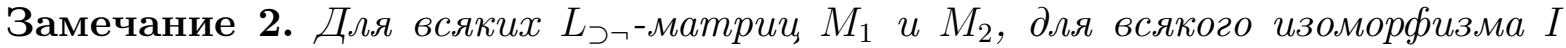

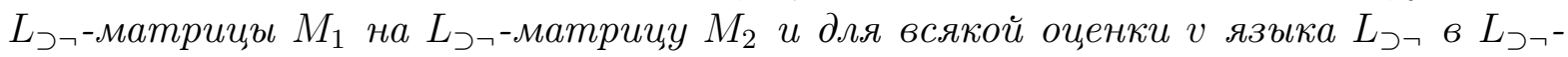
матрище $M_{1}$ существует единственное множество $I[v]$ всех упорядоченных пар, каждая из которых имеет вид $\langle q, x\rangle$, где q есть пропозищиональная переменная

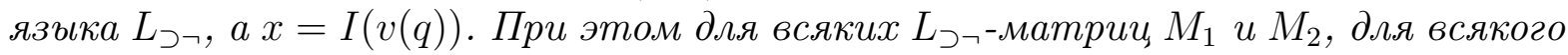

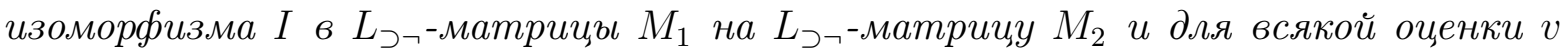

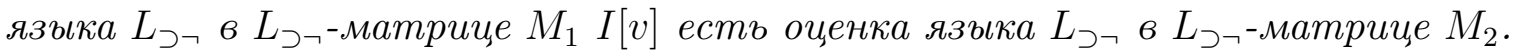

Вспомним замечание 2 из (Попов 2019a), согласно которому для всякой $L_{\supset\urcorner^{-}}$ матрицы $K$ существует единственное отображение (обозначим его через $\varphi_{K}$ ) множества всех упорядочнных пар, каждая из которых имеет вид $\langle A, w\rangle$, где $A$ есть

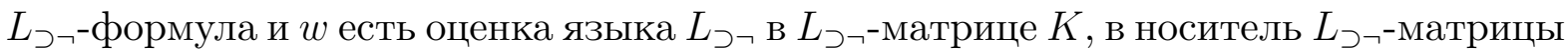
$K$, выполняющее следующие три условия: (1) для всякой пропозициональной переменной $q$ языка $L_{\supset \neg}$ и для всякой оценки $v$ языка $L_{\supset \neg}$ в $L_{\supset}$-матрице $K$ верно,

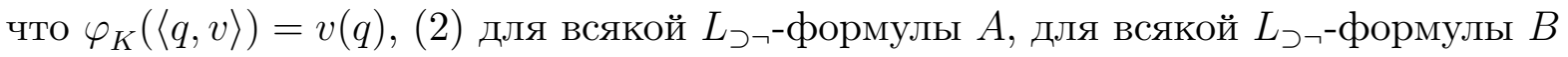

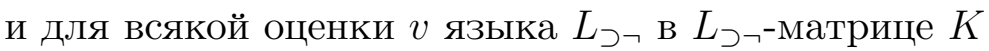

$$
\varphi_{K}(\langle(A \supset B), v\rangle)=\left(\varphi_{K}(\langle A, v\rangle) g \varphi_{K}(\langle B, v\rangle)\right),
$$

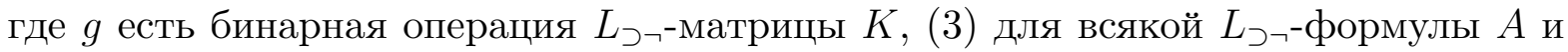

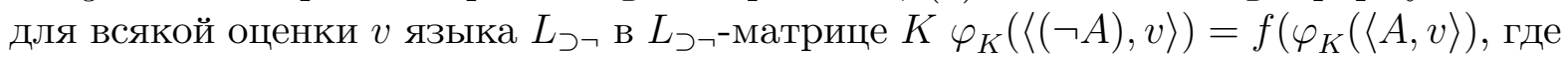
$f$ есть бинарная операция $L_{\supset \neg}$-матрицы $K$.

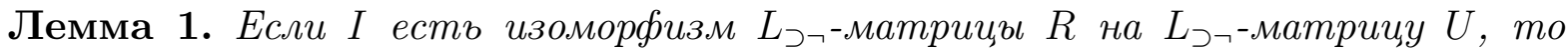

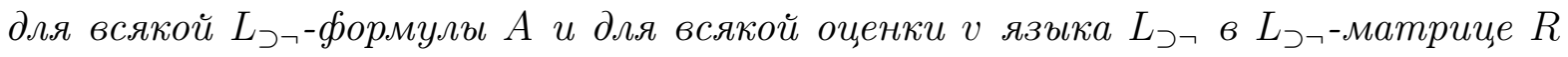
$I\left(\varphi_{R}(\langle A, v\rangle)\right)=\varphi_{U}(\langle A, I[v]\rangle)$. 
Лемма 1 доказана индукцией по построению $L_{\supset-}$-формулы.

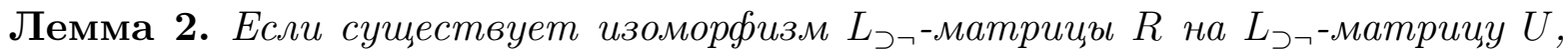
то для всякой $L_{\supset \neg-}$ бормуль $A$ : если $A$ есть $L_{\supset \neg-}$ формула, общезначимая в $L_{\supset \neg^{-}}$

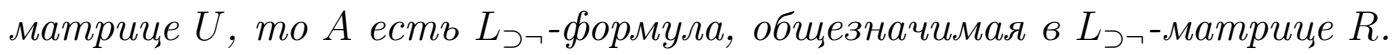

Лемма 2 доказана с использованием леммы 1.

Нижеследующая лемма 3 является «заимствованием» из теории алгебраических систем.

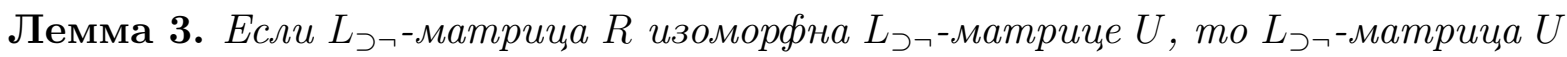

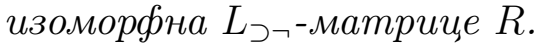

Используя леммы 2 и 3, получаем следующую теорему 1.

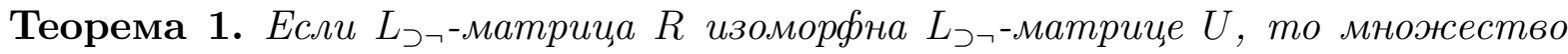

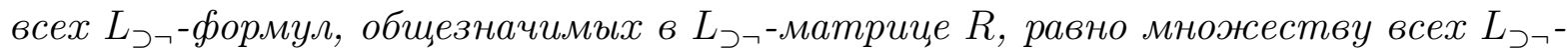

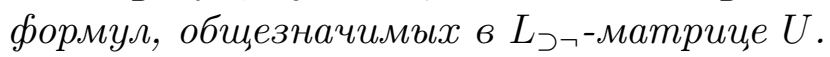

Теорема 2. Для всякой унарной операции $f$ на множестве $\{1,1 / 2,0\}$ упорядоченная пара $\langle M(1 / 2,1,0,1 / 2), f\rangle$ есть такая $L_{\supset}$-матрица, что множество всех

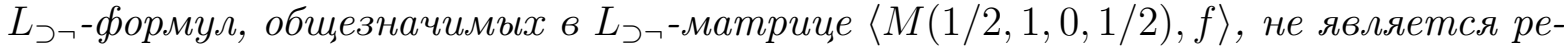

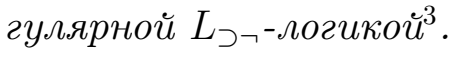

Докажем теорему 2 методом от противного.

(1) Неверно, что для всякой унарной операции $f$ на множестве $\{1,1 / 2,0\}$ упорядоченная пара $\langle M(1 / 2,1,0,1 / 2), f\rangle$ есть такая $L_{\supset \neg}$-матрица, что множество всех

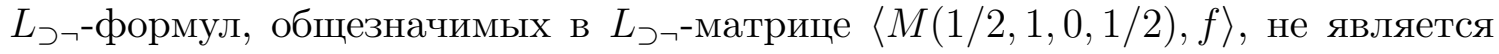
регулярной $L_{\supset\urcorner}$-логикой (допущение).

(2) Для некоторой унарной операции $f$ на множестве $\{1,1 / 2,0\}$ упорядоченная пара $\langle M(1 / 2,1,0,1 / 2), f\rangle$ есть такая $L_{\supset \neg}$-матрица, что множество всех $L_{\supset \neg}$

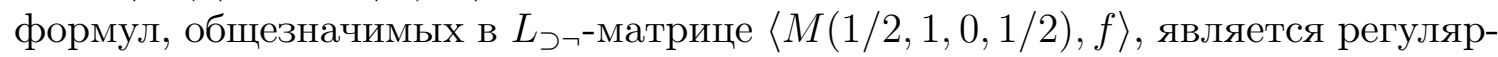

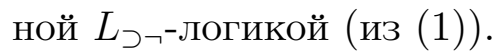

(3) Пусть $f_{0}$ есть унарная операция на множестве $\{1,1 / 2,0\}$ и упорядоченная пара

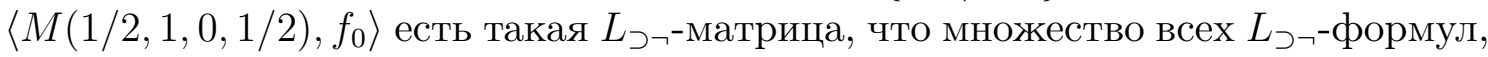
общезначимых в $L_{\supset \neg}$-матрице $\left\langle M(1 / 2,1,0,1 / 2), f_{0}\right\rangle$, является регулярной $L_{\supset}$ логикой.

(4) $f_{0}$ есть унарная операция на множестве $\{1,1 / 2,0\}$ (из (3)).

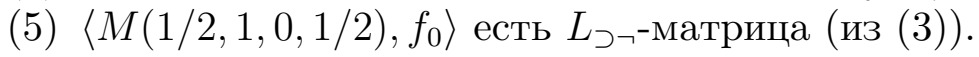

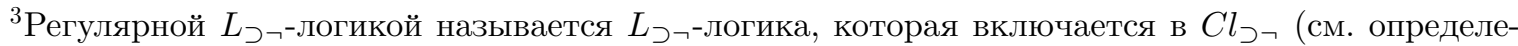
ние 9 из (Попов 2019а)).
} 
Владимир Попов. К проблеме расширения матричной семантики...

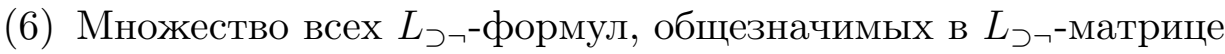

$$
\left\langle M(1 / 2,1,0,1 / 2), f_{0}\right\rangle
$$

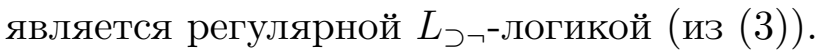

В силу соглашения 7 из (Попов 2019а) верно, что

(7) $M(1 / 2,1,0,1 / 2)=\langle\{1,1 / 2,0\},\{1\}, \supset(1 / 2,1,0,1 / 2)\rangle$.

Опираясь на утверждение 7 , делаем вывод, что

(8) $\left\langle M(1 / 2,1,0,1 / 2), f_{0}\right\rangle=\left\langle\{1,1 / 2,0\},\{1\}, \supset(1 / 2,1,0,1 / 2), f_{0}\right\rangle$.

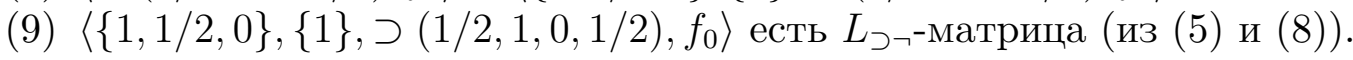

Следуя соглашению 5 из (Попов 2019а), имеем, что

(10) $s$ есть отображение множества $\{1,1 / 2,0\}$ на себя, определяемое таблицей

Ясно, что

$$
\begin{array}{c|ccc}
s & 1 & 1 / 2 & 0 \\
\hline & 1 & 0 & 1 / 2
\end{array}
$$

(11) $\left\{\left\langle 1, s\left(f_{0}(s(1))\right)\right\rangle,\left\langle 1 / 2, s\left(f_{0}(s(1 / 2))\right)\right\rangle,\left\langle 0, s\left(f_{0}(s(0))\right)\right\rangle\right\}$ есть унарная операция на множестве $\{1,1 / 2,0\}$.

Условимся, что

(12) $\left\{\left\langle 1, s\left(f_{0}(s(1))\right)\right\rangle,\left\langle 1 / 2, s\left(f_{0}(s(1 / 2))\right)\right\rangle,\left\langle 0, s\left(f_{0}(s(0))\right)\right\rangle\right\}=f^{\prime}$.

Таким образом,

(13) для всякого $x$ из $\{1,1 / 2,0\} f^{\prime}(x)=s\left(f_{0}(s(x))\right)$.

Учитывая, что $\supset(1 / 2,1,0,1 / 2)$ есть бинарная операция на непустом множестве $\{1,1 / 2,0\}$, включающем множество $\{1\}$, и опираясь на утверждения (11) и (12), получаем по определению 2 из (Попов 2019а), что

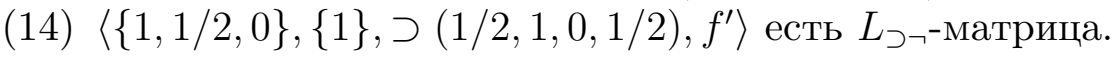

Ясно, что

(15) $s(1)=1$.

Легко проверить, проведя рассуждение по случаям, что

(16) для всякого $x$ из $\{1,1 / 2,0\}$ и для всякого $y$ из $\{1,1 / 2,0\}$

$$
s((x \supset(1 / 2,1,0,1 / 2) y))=(s(x) \supset(1,0,0,1 / 2) s(y)) .
$$

Докажем, что

(17) для всякого $x$ из $\{1,1 / 2,0\} s\left(f^{\prime}(x)\right)=f_{0}(s(x))$.

Доказательство.

(17.1) $x_{0} \in\{1,1 / 2,0\}$ (допущение).

$(17.2) s\left(x_{0}\right) \in\{1,1 / 2,0\}$ (из (17.1) и (10)).

(17.3) $f^{\prime}\left(s\left(x_{0}\right)\right)=s\left(f_{0}\left(s\left(s\left(x_{0}\right)\right)\right)\right)$ (из (17.2) и $\left.(13)\right)$.

Очевидно, что

(17.4) для всякого $x$ из $\{1,1 / 2,0\} s(s(x))=x$.

Опираясь на утверждения (17.3) и (17.4), получаем, что

(17.5) $s\left(f_{0}\left(x_{0}\right)\right)=f^{\prime}\left(s\left(x_{0}\right)\right)$. 
Снимая допущение (17.1) и обобщая, завершаем доказательство утверждения (17).

Утверждение (17) доказано.

Опираясь на утверждения (9), (10), (14), (15), (16), (17) и на определение 1, делаем вывод, что

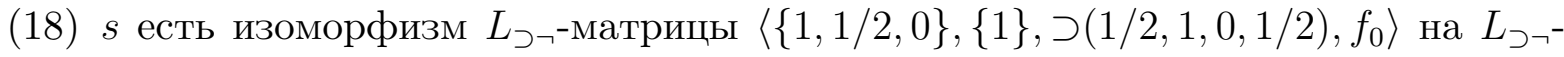
матрицу $\left\langle\{1,1 / 2,0\},\{1\}, \supset(1,0,0,1 / 2), f^{\prime}\right\rangle$.

Из утверждения (18), используя определение 2 , делаем вывод, что

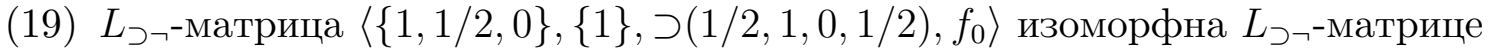

$$
\left\langle\{1,1 / 2,0\},\{1\}, \supset(1,0,0,1 / 2), f^{\prime}\right\rangle .
$$

Опираясь на утверждение (19) и на теорему 1, получаем, что

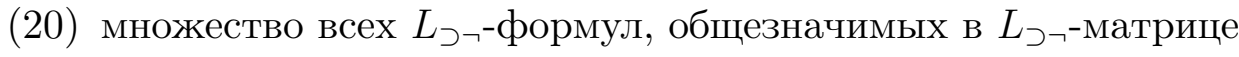

$$
\left\langle\{1,1 / 2,0\},\{1\}, \supset(1 / 2,1,0,1 / 2), f_{0}\right\rangle,
$$

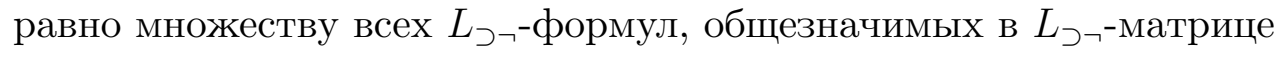

$$
\left\langle\{1,1 / 2,0\},\{1\}, \supset(1,0,0,1 / 2), f^{\prime}\right\rangle .
$$

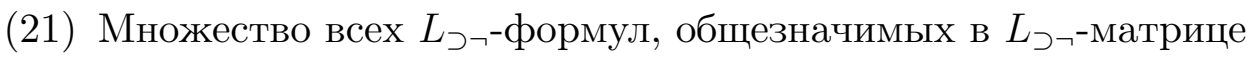

$$
\left\langle\{1,1 / 2,0\},\{1\}, \supset(1 / 2,1,0,1 / 2), f_{0}\right\rangle
$$

является регулярной $L_{\supset-}$-логикой (из (6) и $\left.(9)\right)$.

(22) Множество всех $L_{\supset \neg}$-формул, общезначимых в $L_{\supset \neg}$-матрице

$$
\left\langle\{1,1 / 2,0\},\{1\}, \supset(1,0,0,1 / 2), f^{\prime}\right\rangle
$$

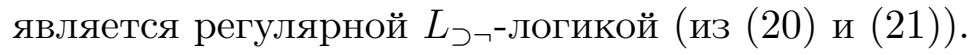

(23) Существует такая унарная операция $f$ на множестве $\{1,1 / 2,0\}$, что множе-

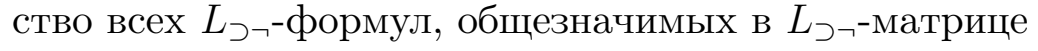

$$
\langle\{1,1 / 2,0\},\{1\}, \supset(1,0,0,1 / 2), f\rangle,
$$

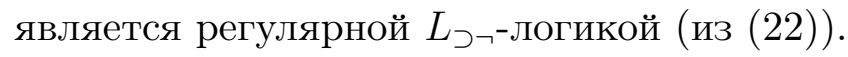

Используя теорему 2 из (Попов 2019а), получаем, что

(24) для всяко унарной операции $f$ на множестве $\{1,1 / 2,0\}$, что множество всех

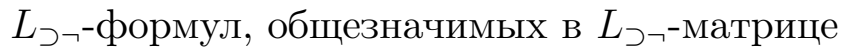

$$
\langle\{1,1 / 2,0\},\{1\}, \supset(1,0,0,1 / 2), f\rangle,
$$

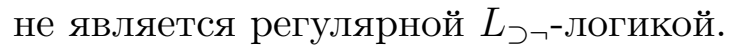

Утверждение (24) противоречит утверждению (23). Следовательно, неверно допущение (1). 
Владимир Попов. К проблеме расширения матричной семантики...

Итак, теорема 2 доказана методом от противного.

В свете теоремы 2 ясно, что не существует логическая матрица вида

$$
\langle M(1 / 2,1,0,1 / 2), f\rangle
$$

(где $f$ есть унарная операция на $\{1,1 / 2,0\}$ ), адекватная классической импликативно-негативной логике $C l_{\supset \neg}$.

\section{Второй раздел}

Теорема 3. Для всякой унарной операции $f$ на множестве $\{1,1 / 2,0\}$

$$
\langle M(1 / 2,0,0,1 / 2), f\rangle
$$

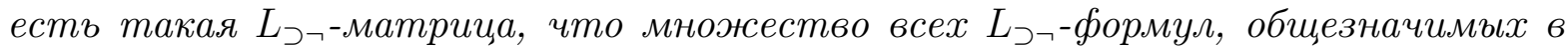

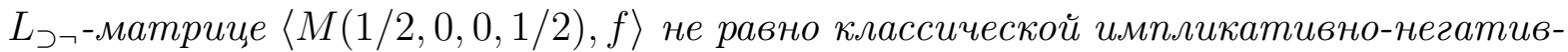
ной логике $C l_{\supset \neg}$.

Докажем теорему 3.

(1) $f_{0}$ есть унарная операция на множестве $\{1,1 / 2,0\}$ (допущение).

Используя замечание 9 из (Попов 2019а) и соглашение 13 из (Попов 2019а), можно доказать, что

(2) для всякого $i: i$ есть унарная операция на множестве $\{1,1 / 2,0\}$ тогда и только тогда, когда верно следующее: $i$ есть $\neg(1, y, z)$, где $y, z \in\{1,1 / 2,0\}$, или $i$ есть $\neg(1 / 2,1, z)$, где $z \in\{1,1 / 2,0\}$, или $i$ есть $\neg(1 / 2,1 / 2, z)$, где $z \in\{1,1 / 2,0\}$, или $i$ есть $\neg(1 / 2,0,1)$, или $i$ есть $\neg(1 / 2,0,1 / 2)$, или $i$ есть $\neg(1 / 2,0,0)$, или $i$ есть $\neg(0,1,1)$, или $i$ есть $\neg(0,1,1 / 2)$, или $i$ есть $\neg(0,1,0)$, или $i$ есть $\neg(0,1 / 2,1)$, или $i$ есть $\neg(0,1 / 2,1 / 2)$, или $i$ есть $\neg(0,1 / 2,0)$, или $i$ есть $\neg(0,0,1)$, или $i$ есть $\neg(0,0,1 / 2)$, или $i$ есть $\neg(0,0,0)$.

(3) $f_{0}$ есть $\neg(1, y, z)$, где $y, z \in\{1,1 / 2,0\}$, или $f_{0}$ есть $\neg(1 / 2,1, z)$, где $z \in\{1,1 / 2,0\}$, или $f_{0}$ есть $\neg(1 / 2,1 / 2, z)$, где $z \in\{1,1 / 2,0\}$, или $f_{0}$ есть $\neg(1 / 2,0,1)$, или $f_{0}$ есть $\neg(1 / 2,0,1 / 2)$, или $f_{0}$ есть $\neg(1 / 2,0,0)$, или $f_{0}$ есть $\neg(0,1,1)$, или $f_{0}$ есть $\neg(0,1,1 / 2)$, или $f_{0}$ есть $\neg(0,1,0)$, или $f_{0}$ есть $\neg(0,1 / 2,1)$, или $f_{0}$ есть $\neg(0,1 / 2,1 / 2)$, или $f_{0}$ есть $\neg(0,1 / 2,0)$, или $f_{0}$ есть $\neg(0,0,1)$, или $f_{0}$ есть $\neg(0,0,1 / 2)$, или $f_{0}$ есть $\neg(0,0,0)$ (из $(1)$ и $(3))$.

Здесь прервем доказательство теоремы 3 и сделаем следующие замечания 3-17, в справедливости которых нетрудно убедиться, применив простую процедуру проверки общезначимости пропозициональных формул в конечных логических матрицах.

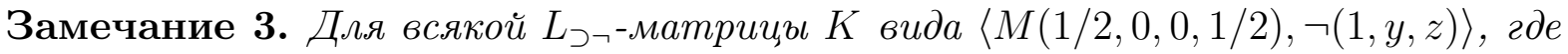
$y, z \in\{1,1 / 2,0\}$, верно, что $\left(\neg\left(p_{1} \supset p_{1}\right)\right)$ есть $L_{\supset \neg}$-формула, общезначимая в $L_{\supset \neg-}$ матрище $K$. 
Замечание 4. Для всякой $L_{\supset \neg-м а т р и ц ь ~} K$ вида $\langle M(1 / 2,0,0,1 / 2), \neg(1 / 2,1, z)\rangle$, где

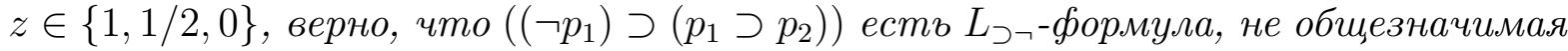

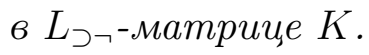

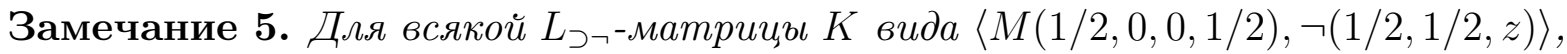

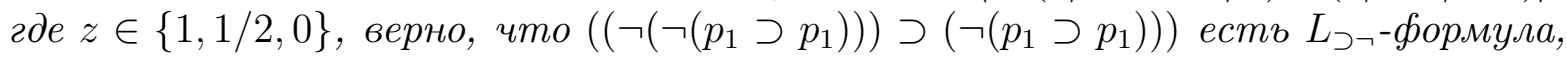

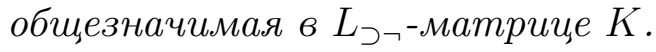

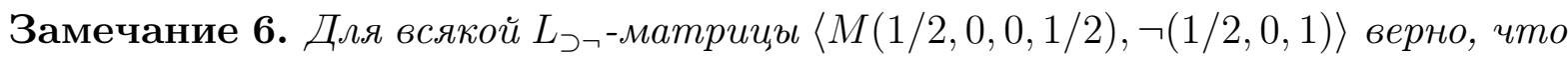
$\left(\left(p_{1} \supset p_{1}\right) \supset\left(\neg\left(\neg\left(\neg\left(p_{1} \supset p_{1}\right)\right)\right)\right)\right)$ есть $L_{\supset \neg^{-}}$бормула, общезначимая в этой $L_{\supset \neg^{-}}$ матриче.

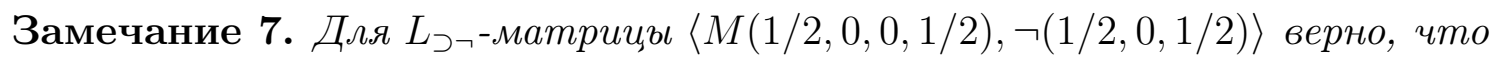

$$
\left(\neg\left(\neg\left(p_{1} \supset p_{1}\right)\right)\right)
$$

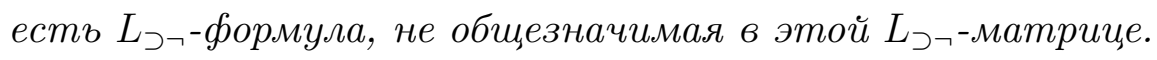

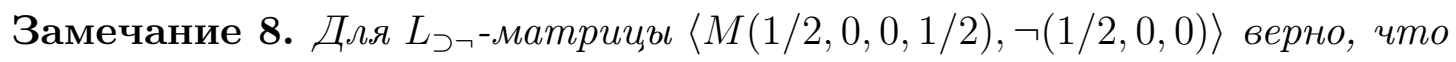

$$
\left(\left(\neg\left(\neg\left(p_{1} \supset p_{1}\right)\right)\right) \supset\left(\neg\left(\neg\left(\neg\left(p_{1} \supset p_{1}\right)\right)\right)\right)\right)
$$

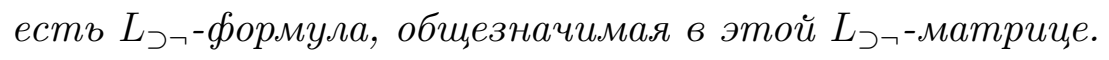

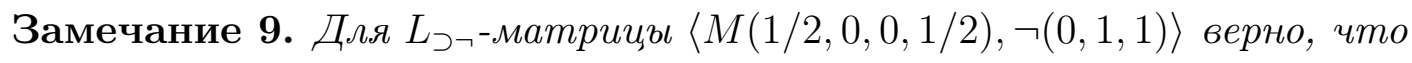

$$
\left(\left(\neg p_{1}\right) \supset\left(p_{1} \supset p_{1}\right)\right)
$$

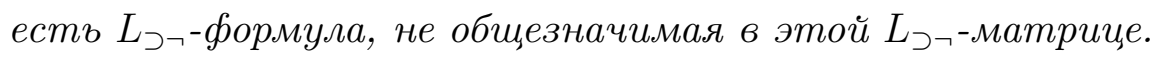

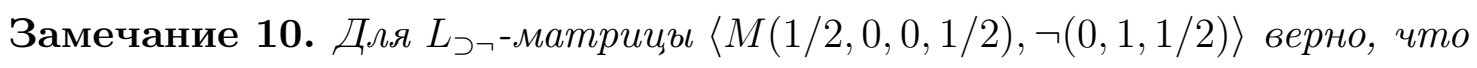

$$
\left(\neg\left(\neg\left(\neg\left(p_{1} \supset p_{1}\right)\right)\right)\right)
$$

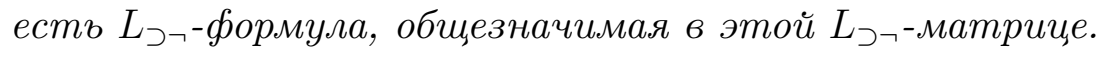

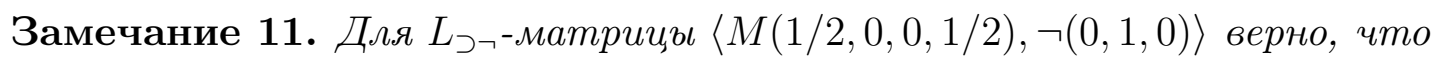

$$
\left(\left(\neg\left(\neg\left(p_{1} \supset p_{1}\right)\right)\right) \supset\left(\neg\left(p_{1} \supset p_{1}\right)\right)\right)
$$

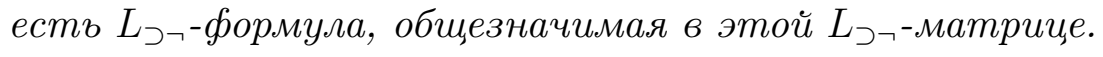

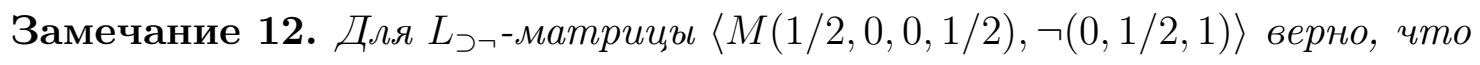

$$
\left(\left(\neg p_{1}\right) \supset\left(p_{1} \supset p_{2}\right)\right)
$$

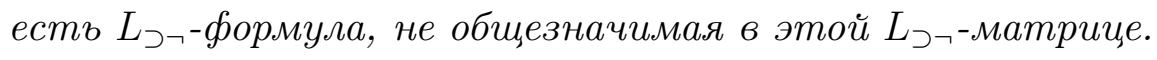


Владимир Попов. К проблеме расширения матричной семантики...

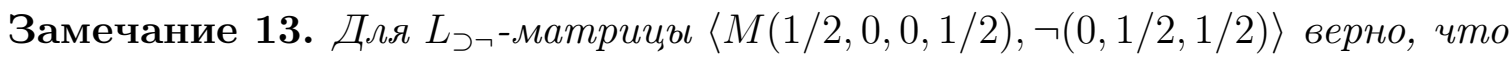

$$
\left(\left(\neg\left(\neg\left(p_{1} \supset p_{1}\right)\right)\right) \supset\left(\neg\left(\neg\left(\neg\left(p_{1} \supset p_{1}\right)\right)\right)\right)\right)
$$

есть $L_{\supset \neg-}$ бормула, общезначимая в этой $L_{\supset\urcorner}$-матрище.

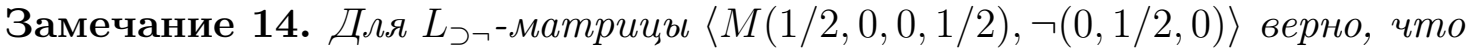

$$
\left(\left(\neg\left(\neg\left(p_{1} \supset p_{1}\right)\right)\right) \supset\left(\neg\left(p_{1} \supset p_{1}\right)\right)\right)
$$

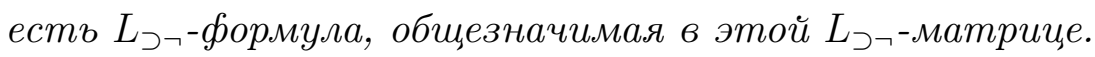

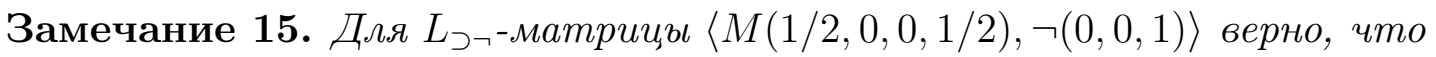

$$
\left(\left(\neg p_{1}\right) \supset\left(p_{1} \supset p_{2}\right)\right)
$$

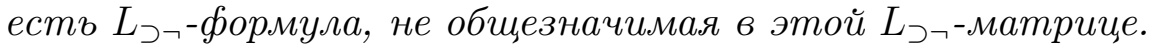

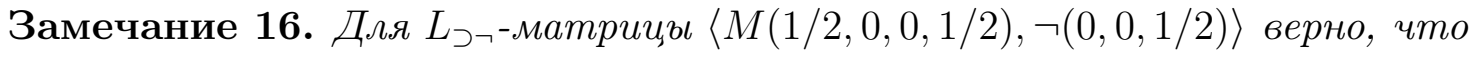

$$
\left(\neg\left(\neg\left(p_{1} \supset p_{1}\right)\right)\right)
$$

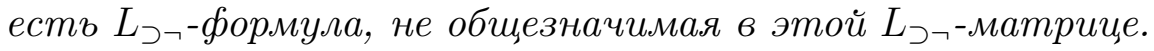

Замечание 17. Для $L_{\supset \neg-м а т р и щ ы ~}\langle M(1 / 2,0,0,1 / 2), \neg(0,0,0)\rangle$ верно, что

$$
\left(\left(\neg\left(\neg\left(p_{1} \supset p_{1}\right)\right)\right) \supset\left(\neg\left(p_{1} \supset p_{1}\right)\right)\right)
$$

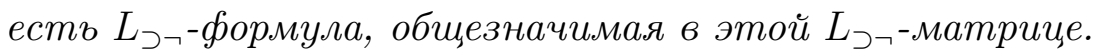

Вернемся к доказательству теоремы 3.

Опираясь на замечание 3 и тот факт, что $\left(\neg\left(p_{1} \supset p_{1}\right)\right)$ есть $L_{\supset}$-формула, не общезначимая в $M\left(C l_{\supset \neg}\right)$, получаем, что

(4) если $f_{0}$ есть $\neg(1, y, z)$, где $x, y \in\{1,1 / 2,0\}$, то $\left\langle M(1 / 2,0,0,1 / 2), f_{0}\right\rangle$ есть такая

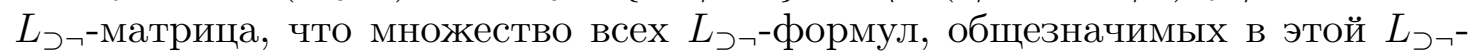
матрице, не равно классической импликативно-негативной логике $C l_{\supset \neg}$.

Опираясь на замечание 4 и тот факт, что $\left(\left(\neg p_{1}\right) \supset\left(p_{1} \supset p_{2}\right)\right)$ есть $L_{\supset \neg}$

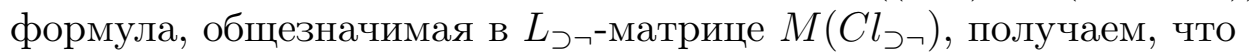

(5) если $f_{0}$ есть $\neg(1 / 2,1, z)$, где $z \in\{1,1 / 2,0\}$, то $\left\langle M(1 / 2,0,0,1 / 2), f_{0}\right\rangle$ есть такая $L_{\supset \neg}$-матрица, что множество всех $L_{\supset \neg}$-формул, общезначимых в этой $L_{\supset \neg}$ матрице, не равно классической импликативно-негативной логике $C l_{\supset \neg}$.

Опираясь на замечание 5 и тот факт, что $\left(\left(\neg\left(\neg\left(p_{1} \supset p_{1}\right)\right)\right) \supset\left(\neg\left(p_{1} \supset p_{1}\right)\right)\right)$ есть $L_{\supset \neg}$-формула, не общезначимая в $L_{\supset \neg-м а т р и ц е ~} M\left(C l_{\supset \neg}\right)$, получаем, что 
(6) если $f_{0}$ есть $\neg(1 / 2,1 / 2, z)$, где $z \in\{1,1 / 2,0\}$, то $\left\langle M(1 / 2,0,0,1 / 2), f_{0}\right\rangle$ есть такая $L_{\supset \dashv}$-матрица, что множество всех $L_{\supset-}$-формул, общезначимых в этой $L_{\supset \neg}$-матрице, не равно классической импликативно-негативной логике $C l_{\supset \neg}$. Опираясь на замечание 6 и тот факт, что $\left(\left(p_{1} \supset p_{1}\right) \supset\left(\neg\left(\neg\left(\neg\left(p_{1} \supset p_{1}\right)\right)\right)\right)\right)$

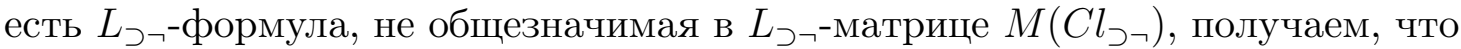

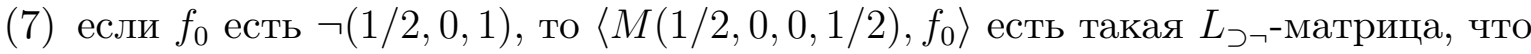

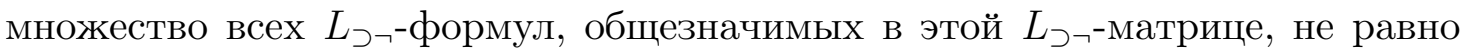
классической импликативно-негативной логике $C l_{\supset \neg}$.

Опираясь на замечание 7 и тот факт, что $\left(\neg\left(\neg\left(p_{1} \supset p_{1}\right)\right)\right)$ есть $L_{\supset \neg-}$-формула, общезначимая в $L_{\supset \neg}$-матрице $M\left(C l_{\supset \neg}\right)$, получаем, что

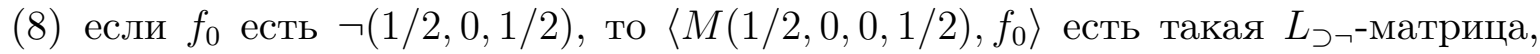

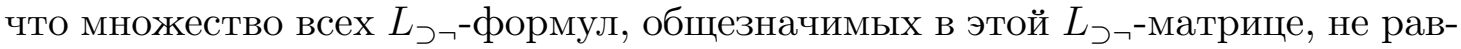
но классической импликативно-негативной логике $C l_{\supset \neg}$.

Опираясь на замечание 8 и тот факт, что $\left(\left(\neg\left(\neg\left(p_{1} \supset p_{1}\right)\right)\right) \supset\left(\neg\left(\neg\left(\neg\left(p_{1} \supset\right.\right.\right.\right.\right.$ $\left.\left.\left.\left.p_{1}\right)\right)\right)\right)$ ) есть $L_{\supset \neg}$-формула, не общезначимая в $L_{\supset \neg-м а т р и ц е ~} M\left(C l_{\supset \neg}\right)$, получаем, что

(9) если $f_{0}$ есть $\neg(1 / 2,0,0)$, то $\left\langle M(1 / 2,0,0,1 / 2), f_{0}\right\rangle$ есть такая $L_{\supset \dashv}$-матрица, что

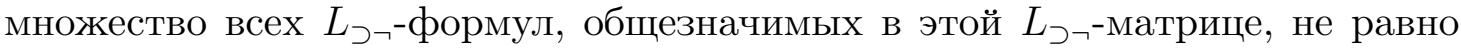
классической импликативно-негативной логике $\mathrm{Cl}_{\supset \text { ᄀ. }}$.

Опираясь на замечание 9 и тот факт, что $\left(\left(\neg p_{1}\right) \supset\left(p_{1} \supset p_{2}\right)\right)$ есть $L_{\supset\urcorner^{-}}$ формула, общезначимая в $L_{\supset \neg}$-матрице $M\left(C l_{\supset \neg}\right)$, получаем, что

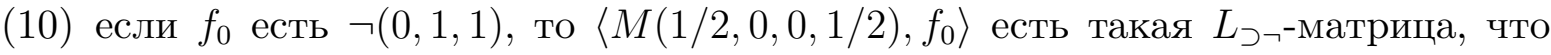
множество всех $L_{\supset \dashv}$-формул, общезначимых в этой $L_{\supset-}$-матрице, не равно классической импликативно-негативной логике $C l_{\supset \neg}$.

Опираясь на замечание 10 и тот факт, что $\left(\neg\left(\neg\left(\neg\left(p_{1} \supset p_{1}\right)\right)\right)\right)$ есть $L_{\supset\urcorner^{-}}$

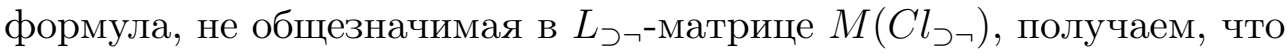

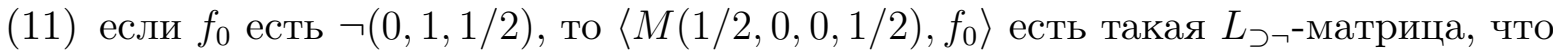

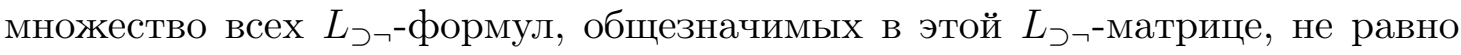
классической импликативно-негативной логике $C l_{\supset \neg}$.

Опираясь на замечание 11 и тот факт, что $\left(\left(\neg\left(\neg\left(p_{1} \supset p_{1}\right)\right)\right) \supset\left(\neg\left(p_{1} \supset p_{1}\right)\right)\right)$

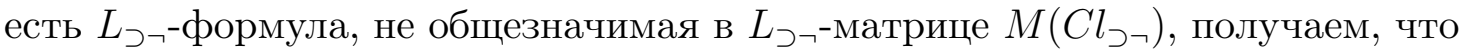

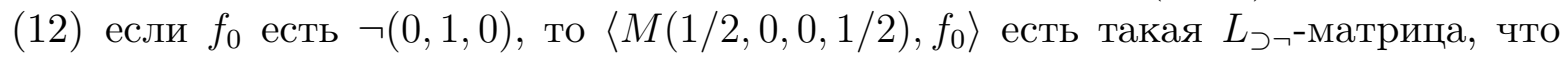

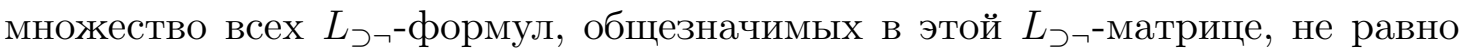
классической импликативно-негативной логике $C l_{\supset \neg}$.

Опираясь на замечание 12 и тот факт, что $\left(\left(\neg p_{1}\right) \supset\left(p_{1} \supset p_{2}\right)\right)$ есть $L_{\supset\urcorner^{-}}$

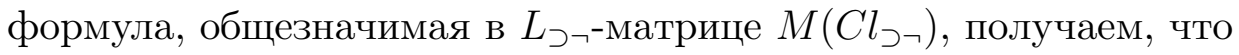

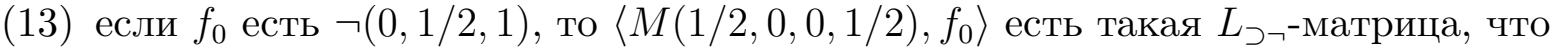

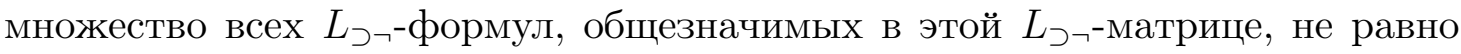
классической импликативно-негативной логике $\mathrm{Cl}_{\supset \text { ᄀ. }}$.

Опираясь на замечание 13 и тот факт, что $\left(\left(\neg\left(\neg\left(p_{1} \supset p_{1}\right)\right)\right) \supset\left(\neg\left(\neg\left(\neg\left(p_{1} \supset\right.\right.\right.\right.\right.$

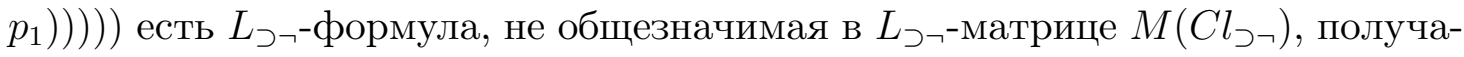


Владимир Попов. К проблеме расширения матричной семантики...

ем, что

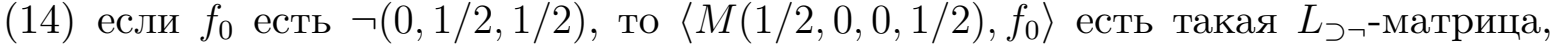
что множество всех $L_{\supset \neg}$-формул, общезначимых в этой $L_{\supset \neg}$-матрице, не равно классической импликативно-негативной логике $C l_{\supset \neg}$.

Опираясь на замечание 14 и тот факт, что $\left(\left(\neg\left(\neg\left(p_{1} \supset p_{1}\right)\right)\right) \supset\left(\neg\left(p_{1} \supset p_{1}\right)\right)\right)$ есть $L_{\supset \neg}$-формула, не общезначимая в $L_{\supset \neg-м а т р и ц е ~} M\left(C l_{\supset \neg}\right)$, получаем, что

(15) если $f_{0}$ есть $\neg(0,1 / 2,0)$, то $\left\langle M(1 / 2,0,0,1 / 2), f_{0}\right\rangle$ есть такая $L_{\supset}$-матрица, что

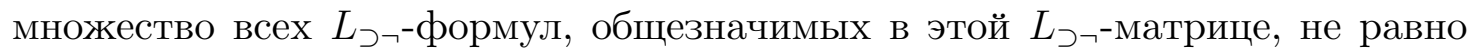
классической импликативно-негативной логике $C l_{\supset \neg}$.

Опираясь на замечание 15 и тот факт, что $\left(\left(\neg p_{1}\right) \supset\left(p_{1} \supset p_{2}\right)\right)$ есть $L_{\supset \neg}$ формула, общезначимая в $L_{\supset \neg}$-матрице $M\left(C l_{\supset \neg}\right)$, получаем, что

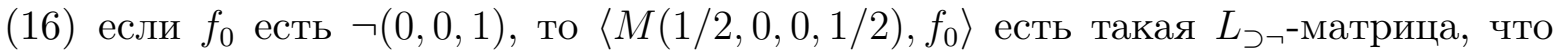

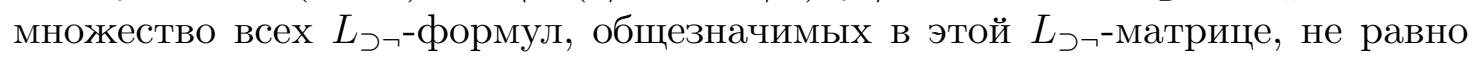
классической импликативно-негативной логике $C l_{\supset \neg}$.

Опираясь на замечание 16 и тот факт, что $\left(\neg\left(\neg\left(p_{1} \supset p_{1}\right)\right)\right)$ есть $L_{\supset \neg}$-формула,

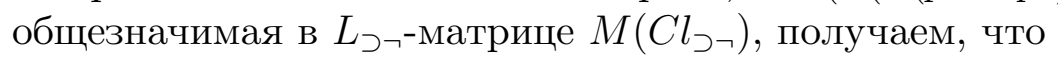

(17) если $f_{0}$ есть $\neg(0,0,1 / 2)$, то $\left\langle M(1 / 2,0,0,1 / 2), f_{0}\right\rangle$ есть такая $L_{\supset}$-матрица, что

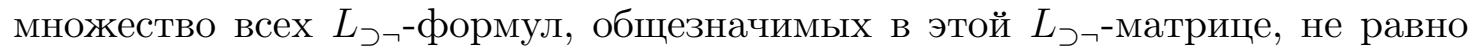
классической импликативно-негативной логике $C l_{\supset \neg}$.

Опираясь на замечание 17 и тот факт, что $\left(\left(\neg\left(\neg\left(p_{1} \supset p_{1}\right)\right)\right) \supset\left(\neg\left(p_{1} \supset p_{1}\right)\right)\right)$

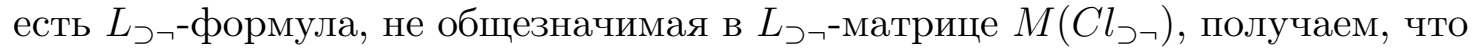

(18) если $f_{0}$ есть $\neg(0,0,0)$, то $\left\langle M(1 / 2,0,0,1 / 2), f_{0}\right\rangle$ есть такая $L_{\supset}$-матрица, что

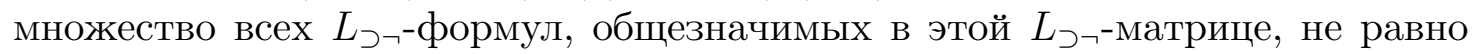
классической импликативно-негативной логике $C l_{\supset \neg}$.

Из утверждений (3)-(18) вытекает, что

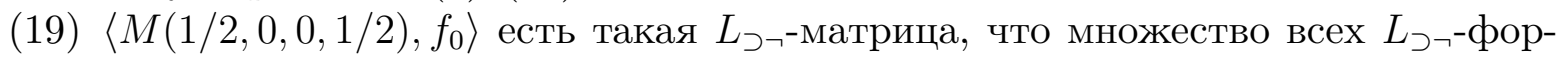
мул, общезначимых в $L_{\supset}$-матрице $\left\langle M(1 / 2,0,0,1 / 2), f_{0}\right\rangle$, не равно классической импликативно-негативной логике $C l_{\supset \neg}$.

Поскольку $f_{0}$ - произвольная унарная операция на множестве $\{1,1 / 2,0\}$ (см. утверждение (1)), проводим обобщение утверждения (19), завершая доказательство теоремы 3.

Теорема 3 доказана.

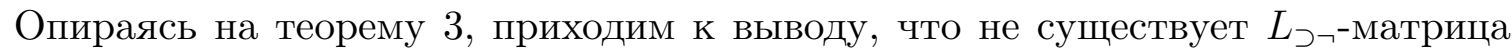
вида $\langle M(1 / 2,0,0,1 / 2), f\rangle$, адекватная классической импликативно-негативной логике $C l_{\supset \neg}$.

Следует заметить, что в свете теоремы 3 очевидно, что анонс, сделанный в (Попов 2019а), требует исправления. Откорректированный анонс гласит:

(i) всякая адекватная классической импликативной логике трехзначная $L_{\supset}$-матрица $K$ с одним выделенным значением, для которой не существует такой унарной операции $f$, что $\langle K, f\rangle$ является $L_{\supset \text { - }}$-матрицей, адекватной классической импликативно-негативной логике, изоморфна $L_{\supset}$-матрице $M(1,0,0,1 / 2)$ 
или изоморфна $L_{\supset}$-матрице $M(1 / 2,0,0,1 / 2)$,

(ii) $\{M(1,0,0,1 / 2), M(1 / 2,1,0,1 / 2), M(1 / 2,0,0,1 / 2)\}$ есть множество всех таких

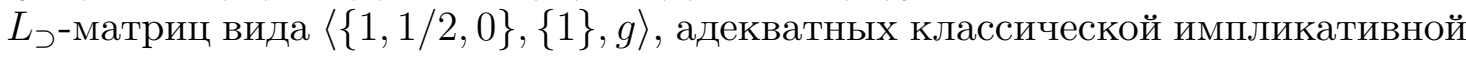
логике, что ни для какой $L_{\supset}$-матрицы $K$ из этого множества $L_{\supset}$-матриц не

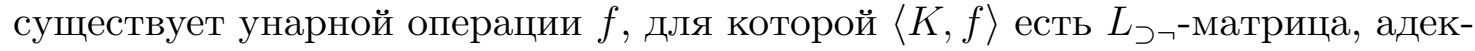
ватная классической импликативно-негативной логике.

\section{Литература}

Попов $2019 \mathrm{a}$ - Попов В. М. К проблеме расширения матричной семантики, адекватной классической импликативной логике, до матричной семантики, адекватной классической импликативно-негативной логике // Логико-философские штудии. 2019. Т. 17, № 1. С. $1-31$.

Попов 2019b - Попов В. М. Трехзначные логические матрицы с одним выделенным значением, адекватные классической импликативной логике // Логико-философские штудии. 2019. Т. 17, № 2. С. 142-193. 